\title{
Vehicle Identification by OCR, RFID and Bluetooth for Toll Roads
}

\author{
Yair Wiseman \\ Computer Science Department, Bar-Ilan University \\ Ramat-Gan 52900, Israel \\ wiseman@cs.biu.ac.il
}

\begin{abstract}
Automatic payment in toll roads can improve the efficiency of the roads; however, errors in this payment can reduce the satisfaction of both the road users and the operators who will both have to waste time in order to sort out these mistakes. It is suggested to greatly minimize these mistakes by backup the recognition course of action of the license plates by using three different methods: RFID, Bluetooth and OCR in order to make recognition errors as seldom as possible.
\end{abstract}

Keywords: Toll Road, RFID, Bluetooth, OCR

\section{Introduction}

Toll roads are usually built in Build-Operate-Transfer (BOT) model, wherein a private commercial business obtains a concession from the government to finance, construct, operate, and collet money from the travelers in a road. This facilitates the road proponent to recover its investment, operating and maintenance expenses in the road [1].

The money collection from the drivers in the toll road can be automatically done by OCR systems which can recognize license plates [2], RFID devices that are installed in the vehicles [3] or Bluetooth equipment that communicates with the apparatus in the road entries [4].

Errors sometimes occur when a vehicle is not identified or when a vehicle is wrongly identified as another vehicle. In such cases the road operator will have a loss. In addition, if a wrong vehicle has been detected, an unhappy driver will be added to the case that will have a hassle in cancelling the wrong bill and a negative advertisement for the service of the toll road can be overstated.

In a few years the vehicles will be autonomous [5], so the need for an embedded system in the vehicle [6,7] for automatic fee collection systems will be even more imperative. Some of the autonomous vehicles will be with neither a driver, nor a passenger, because they will drive alone to a parking lot $[8,9]$, so there will be no one to talk to and the only possible option will be an automatic fee collection system that will charge the fee.

In this paper we suggest to incorporate the 3 ways mentioned above i.e., OCR system, RFID device and Bluetooth equipment as redundant tools in order to get the best results and to overcome mistakes.

Figure 1 shows an RFID tag on a vehicle. The RFID tag was circled and there is an enlargement of it in the right side of the picture.

Received (April 15, 2018), Review Result (June 10, 2018), Accepted (June 17, 2018) 


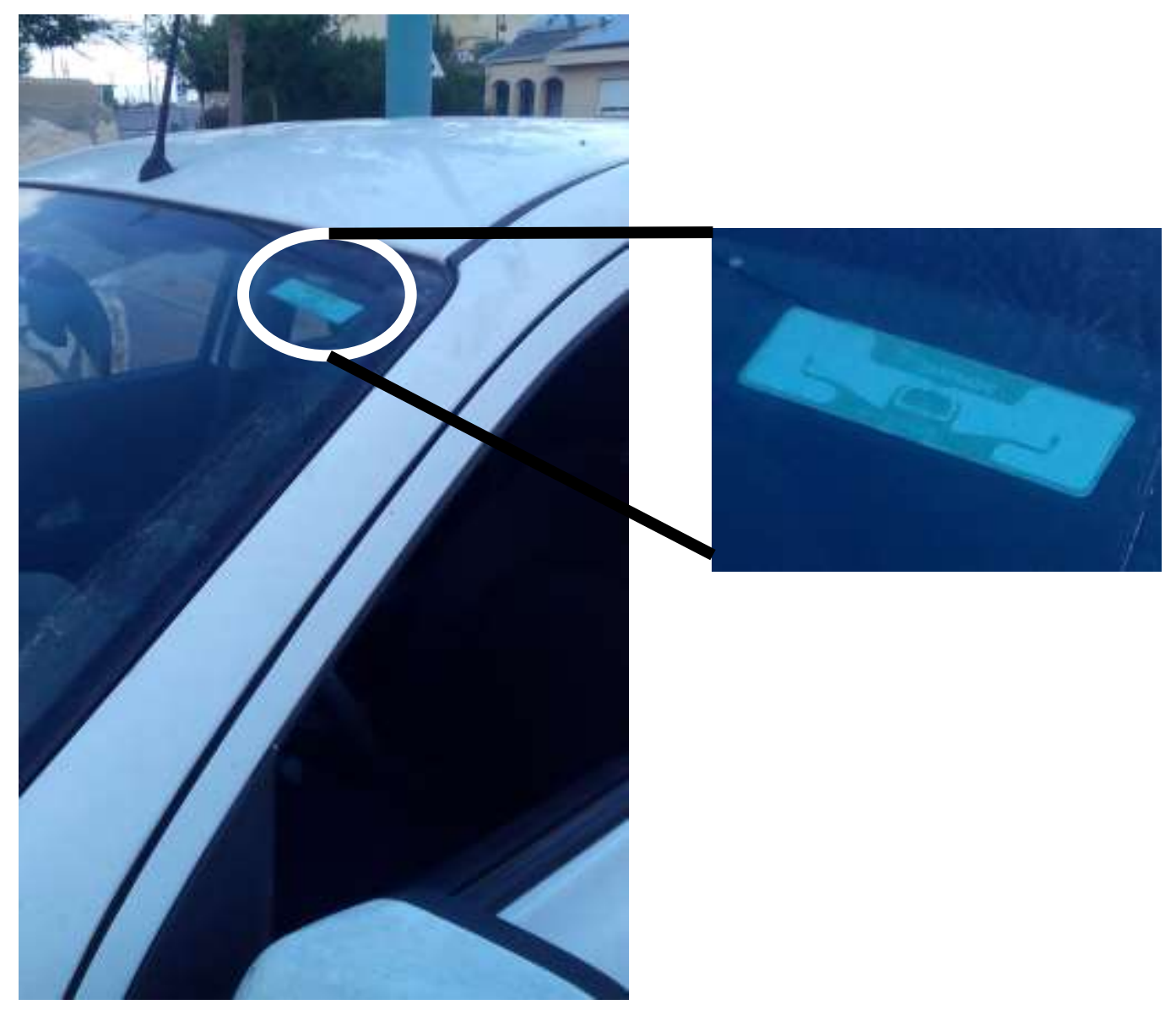

Figure 1. RFID Sticker on a Car

\section{Implementation}

Most of Intelligent Transportation Systems are implemented by state machines [10, 11]. We also used a state machine in order to implement the incorporate license plate recognition device.

RFID is the most efficient method in term of information transformation [12, 13, 14]. In addition, RFID is the most reliable method with an error bit rate of less than $0.001 \%$ [15]. Bluetooth has a higher error bit rate of $0.1 \%$ [16], whereas OCR has the highest error bit rate of $3.6 \%$ [17] because it is an intricate task to decode the information from a picture taken by a camera $[18,19,20]$.

Accordingly, we designed our state machine as described in Figure 2. We gave the RFID and the Bluetooth the first priority. Just if they do not agree, we will check the value of the OCR. 


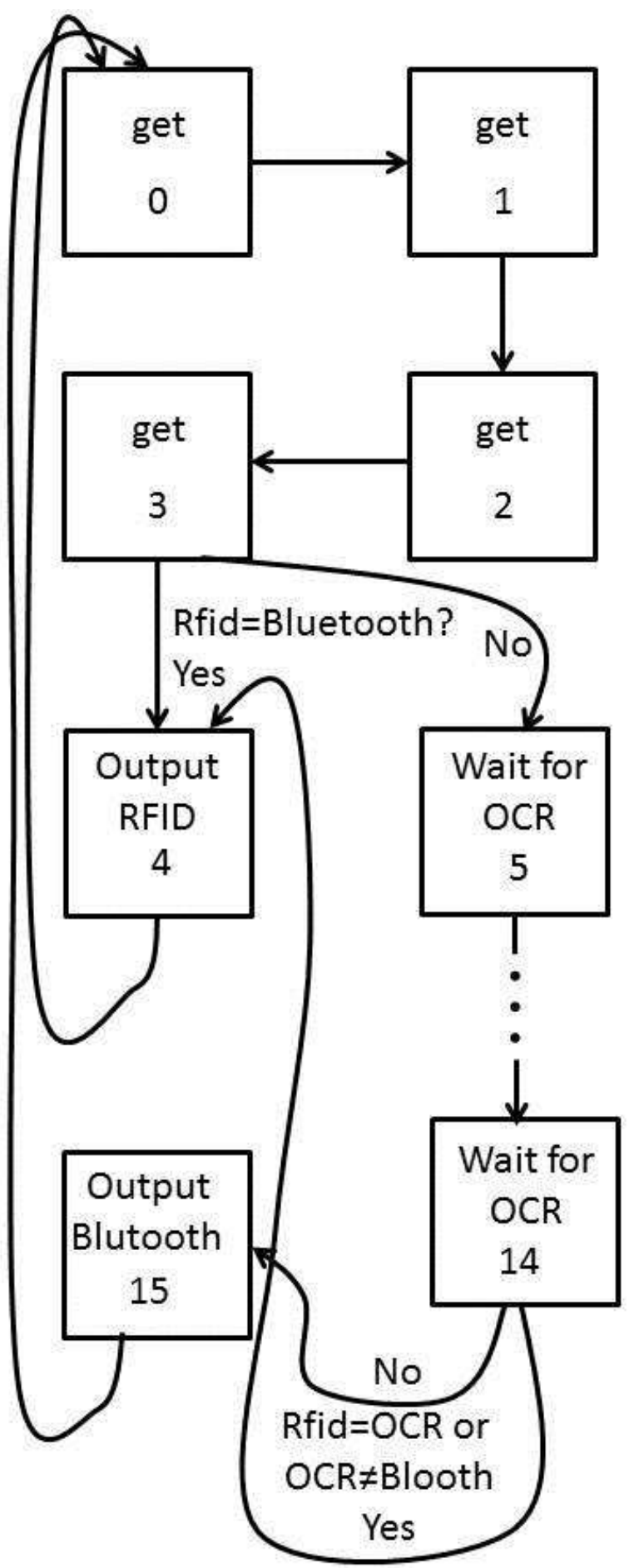

Figure 2. State Machine for the Combined System

The system works as follow: First it gets the value obtained by the RFID and the Bluetooth. Our clock pulse was $0.5 \mathrm{MHz}$ [21]; however, the Bluetooth could not provide the data in less than about $7.4 \mu \mathrm{s}$, so we had to make 4 states of "get" in order to be sure that the data is obtained. It will give us a time of $8 \mu$ s which is enough time to be sure that the Bluetooth data has been obtained. If a faster Bluetooth is available, the four states can be reduced into fewer states.

After obtaining the output of the Bluetooth and the output of the RFID, they will be compared using the comparator circuit described in Figure 3: 


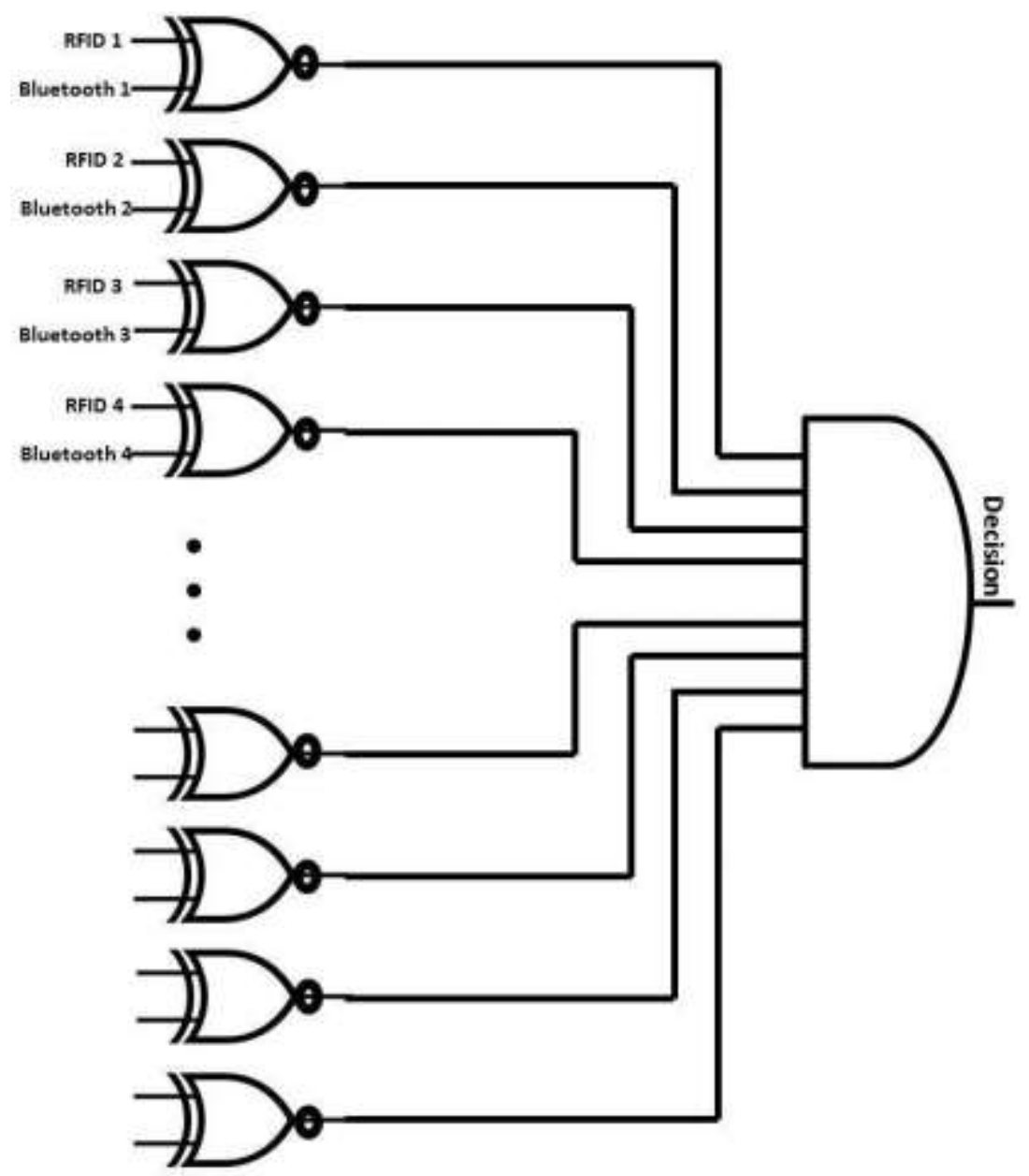

Figure 3. Comparator for RFID and Bluetooth

If the comparator decision is yes, it will denote that the RFID and the Bluetooth agree on the license plate number, this number can be accepted and it will be output as the correct number.

If the comparator decision is no, it will indicate that there is a problem in the RFID or the Bluetooth because they do not come to an agreement what the license plate number is. Then, we will take the OCR as the determining factor; however, the OCR takes more time, that is to say $27.6 \mu$ s, so we will need 14 cycles; however, we can start always to read the OCR in the beginning when the RFID and the Bluetooth begin. If there is no need in the OCR, the OCR can be stopped, but if there is a need in the OCR result, we will have to wait just ten more cycles, because the first four cycles overlap the first four cycles of the Bluetooth and the RFID. The additional ten states are denoted as states 5 to 14 in Figure 2.

If all the devices can come to an agreement, i.e., they produce three different values, the system will take the results of the RFID as this method is the most accurate as was mentioned above.

\section{The Device Output}

The device has several output bits - one bit for indication whether a decision what the license plate number is has been made or the decision has not been made yet. When this indication bit has a value of one, it will indicate that a decision has been made; whereas if this indication bit has a value of zero, it will indicate that no decision has been made yet. 


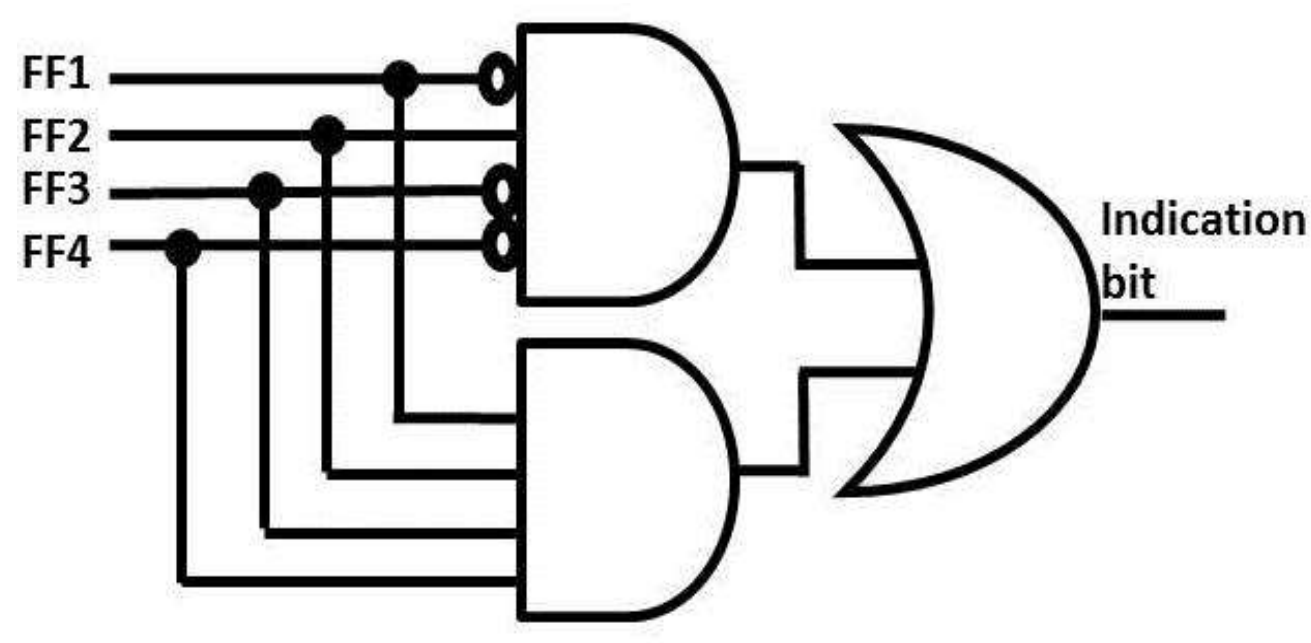

Figure 4. Implementation of the Indication Bit

The other bits are enable bits for the RFID output, the Bluetooth output and the OCR output. When the indication bit denotes that no decision has been made yet, all the enable bits will have a value of zero. When the indication bit changes its value to one, the chosen device will be enabled by setting its bit value to one, whereas the other enable bits will remain with the zero value.

The state machine has 16 states, so it should be implemented with 4 Flip-Flops. The indication bit is set to one just in state 4 and state 5 so the circuit for the indication bit should be implemented as described in Figure 4. In this Figure each one of the Flip-Flops is denoted as FF and it serial number.

There are enable bits actually just to the RFID and the Bluetooth. If the OCR value is equal to the RFID value, the RFID value will be taken. If the OCR value is equal to the Bluetooth value, the Bluetooth value will be taken. If the OCR value is not equal to both the RFID value and the Bluetooth value, the RFID value will be taken, so there is no need for enable bit to the OCR.

The RFID enable bit implementation and the Bluetooth implementation are depicted in Figure 5. The RFID device is enabled only in state 4 and the Bluetooth device is enabled only in state 15 .

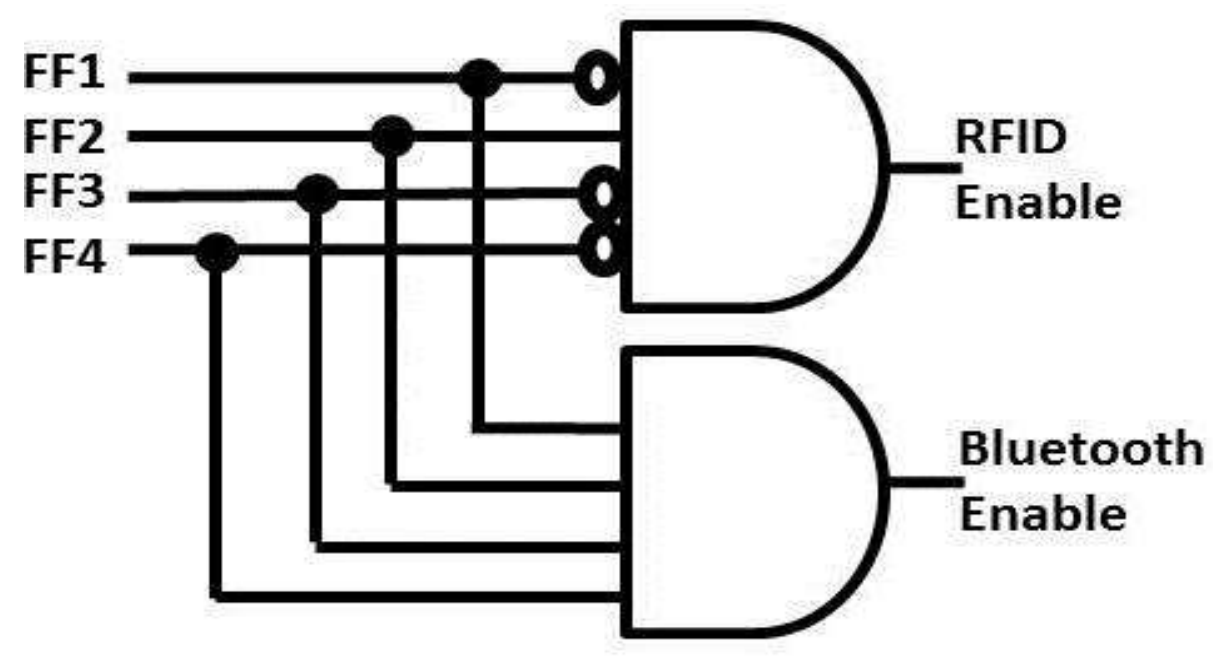

Figure 5. Implementation of the Enable Bits 


\section{The Next State Function}

As was mentioned above, the system has 16 states, so it has 4 Flip-Flops. Accordingly, the system has 4 next state functions for each of the Flip-Flops in the system. We use Karnaugh maps [22,23] to find the simplest functions for implementing the next state circuits. We also assume for simplicity that the implementation uses Edge-Triggered DFilp-Flops [24,25].

The most significantly bit is FF3. If the current state is from 7 to 13 , the next FF3 will be 1 . Also if the current state is 14 and $\mathrm{Rfid} \neq \mathrm{OCR}$ and OCR=Blooth, the next FF3 will be 1. This information is described in the Karnaugh map of Figure 6.

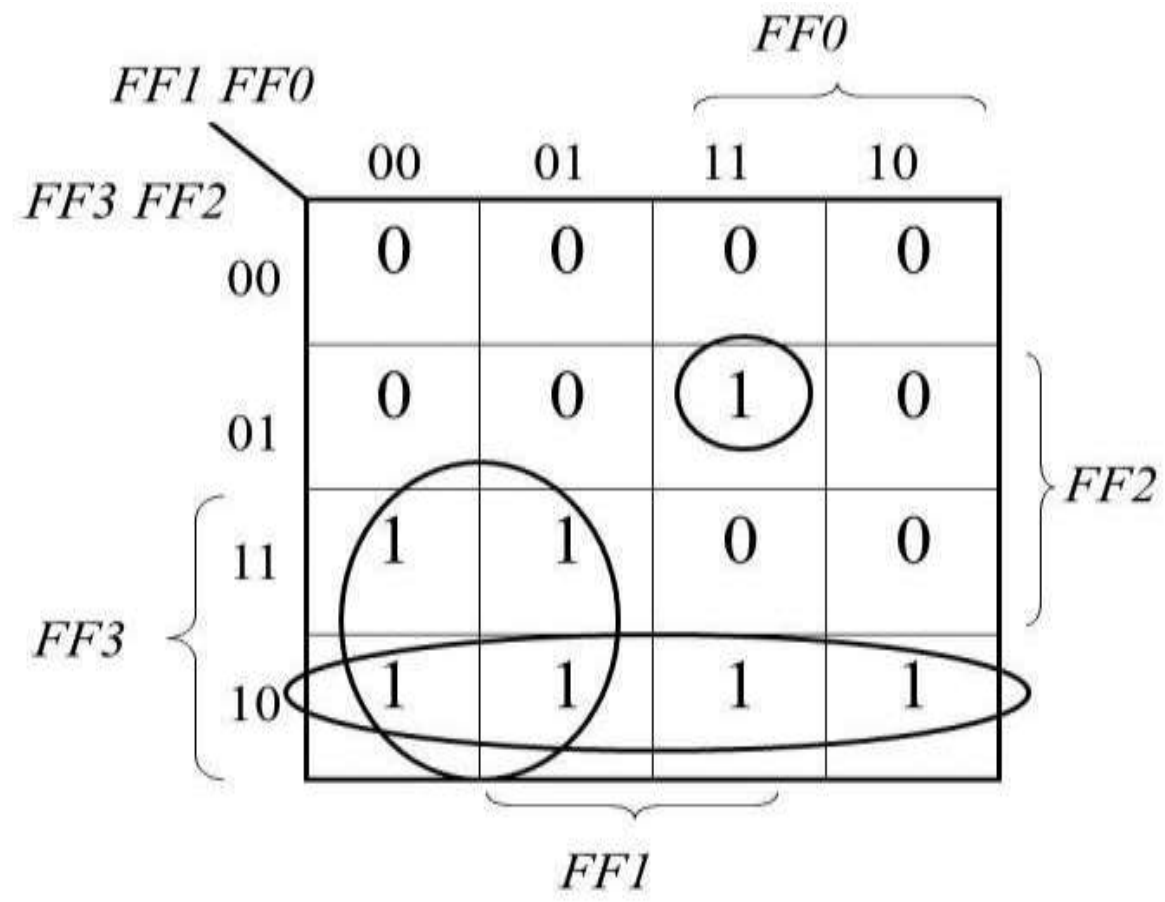

Figure 6. Implementation of Next FF3

According to Figure 6 and according to the condition that if the current state is 14 and Rfid $\neq$ OCR and OCR=Blooth (denoted as condition A), the next FF3 will be 1 , the next state function of FF3 is:

\section{NextFF3=FF3·FF1'+FF3·FF2'+FF3'·FF2'FF1·FF0+(condition A)}

The next state of FF2 has a value of 1 if the current state is 3,5,6,11,12,13,14. Accordingly the Karnaugh map of the next state function of FF2 is described in Figure 7. And the function that is represented by this figure is:

\section{NextFF2=FF2'·FF1·FF0+FF3'FF2'FF1'+FF2'FF1'·FF0+FF2·FF1·FF0'}

It should be noted that the comparators which compare the values of the RFID value, the Bluetooth value and the OCR value do not affect the NextFF2 function. These comparators are the only input to state machine, but they have influence only on the next state function of FF3, FF1 and FF0. 


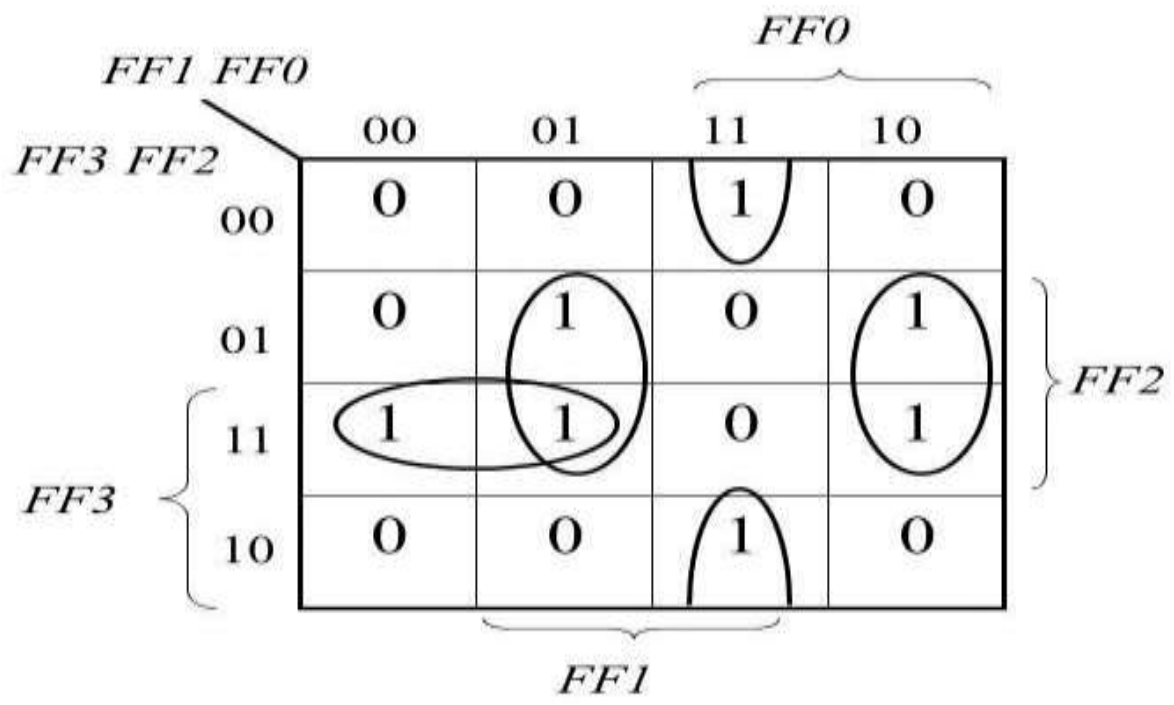

Figure 7. Implementation of Next FF2

NextFF1 is described in Figure 8. NextFF1 will have a value of 1 if the current state is $1,2,5,6,9,10,13$ or the condition that if the current state is 14 and Rfid $\neq$ OCR and OCR=Blooth (denoted as condition A).

Therefore, according to the Karnaugh map of Figure 8 and condition A, the function of NextFF1 is:

\section{NextFF1=FF1'·FF0+FF3'·FF1·FF0+FF2'·FF1·FF0+(condition A)}

The last one is NextFF0 which will have a 1 value if the current state is $0,2,6,8,10,12$. Also, if the current state is 14 and Rfid $\neq$ OCR and OCR=Blooth (denoted as condition A), NextFF0 will be 1 and in addition, if the current state is 3 and Rfid=Bluetooth (denoted as condition B), NextFF0 will be 1 . Subsequently, the function of NextFF0 according to these conditions and according to the Karnaugh map of Figure 9 is:

NextFF0=FF2'·FF0'+FF3·FF1'FF0'+FF3'·FF1·FF0'+(condition A)+(condition B)

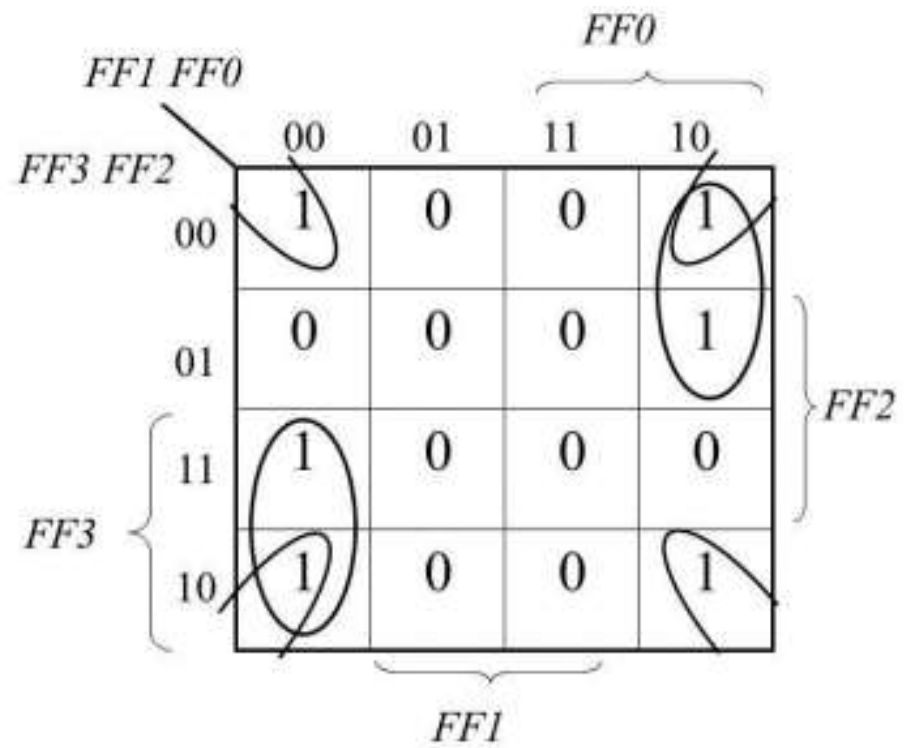

Figure 8. Implementation of Next FF1 


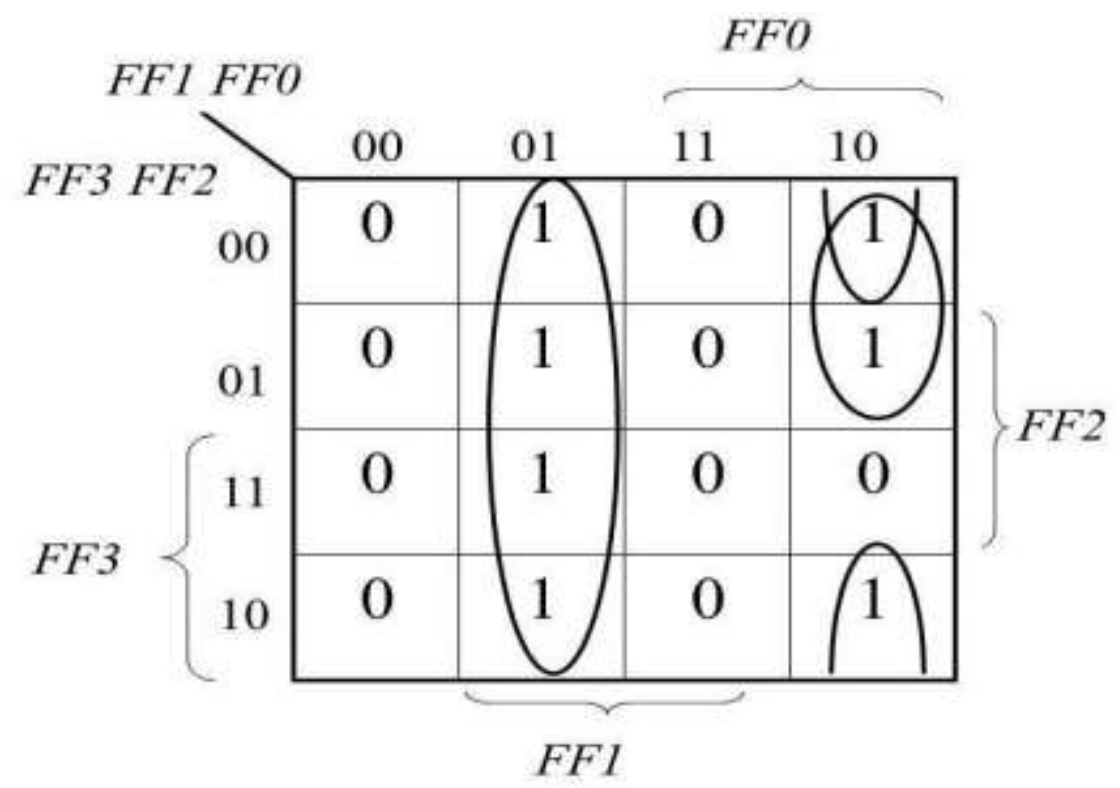

Figure 9. Implementation of Next FF0

\section{Conclusions}

One of the most common areas where Build-Operate-Transfer (BOT) model is carried out is Toll Roads [26, 27, 28]. Our system can help toll road proponents working out more efficient automatic fee collection system. Usually, in new contracts of toll roads, the government requests Intelligent Transportation Systems like collision detection systems [29, 30], so in general toll roads on average have a better safety standard [31]. In addition, toll roads save money of the government budget, because the construction expenses are financed by a private entity. The saved money of the budget can be allocated for alternative projects and other purposes as been done in many countries [32].

The suggested incorporated system can be implemented in a dedicated chip [33] or in a remote manner [34] and can have many benefits for many objectives like better traffic flow [35, 36, 37] or carbon emissions mitigation [38].

\section{References}

[1] H. Yang and M. Qiang, "Highway pricing and capacity choice in a road network under a build-operatetransfer scheme", Transportation Research Part A: Policy and Practice, vol. 34, no. 3, (2000), pp. 207222.

[2] R. M. Khoshki and S. Ganesan, "Improved Automatic License Plate Recognition (ALPR) system based on single pass Connected Component Labeling (CCL) and reign property function", In 2015 IEEE International Conference on Electro/Information Technology (EIT), DeKalb, IL, USA, (2015), pp. 426431.

[3] S. P. Edison, M. Kirubahar, R. Nandhakumar and T. K. Naveenraj, "Electronic Toll Collection Using Portable RFID TAG with Antitheft System", International Journal of Advance Research and Innovation, Paper No. iceict2015i050315004, (2015).

[4] M. A. Sofi and M. Nandhini, "Towards Building smart toll tax collection system using Bluetooth LE Advertising”, International Journal of Advanced Research in Computer Science, vol. 8, no. 1, (2017).

[5] Y. Wiseman, "In an Era of Autonomous Vehicles, Rails are Obsolete", International Journal of Control and Automation, vol. 11, no. 2, (2018), pp. 151-160.

[6] R. Ben Yehuda and Y. Wiseman, "The Offline Scheduler for Embedded Transportation Systems", In Proceedings of IEEE Conference on Industrial Electronics (IEEE ICIT-2011), Auburn, Alabama, (2011), pp. 449-454.

[7] R. Ben Yehuda and Y. Wiseman, "The Offline Scheduler for Embedded Vehicular Systems", International Journal of Vehicle Information and Communication Systems, vol. 3, no. 1, (2013), pp. 4457. 
[8] Y. Wiseman, "Self-Driving Car - A Computer will Park for You", International Journal of Engineering \& Technology for Automobile Security, SERSC publications, vol. 1, no. 1, (2017), pp. 9-16.

[9] Y. Wiseman, "Remote Parking for Autonomous Vehicles", International Journal of Hybrid Information Technology, vol. 10, no. 1, (2017), pp. 313-324.

[10] Y. Wiseman, "Conceptual Design of Intelligent Traffic Light Controller", International Journal of Control and Automation, vol. 9, no. 7, (2016), pp. 251-262.

[11] Y. Wiseman, "Traffic Light with Inductive Detector Loops and Diverse Time Periods", Contemporary Research Trend of IT Convergence Technology, SERSC: Science \& Engineering Research Support society, vol. 4, (2016), pp. 166-170.

[12] Y. Wiseman, "Compaction of RFID Devices using Data Compression", IEEE Journal of Radio Frequency Identification, vol. 1, no. 3, (2017), pp. 202-207.

[13] Y. Wiseman, "Efficient RFID Devices", In Proceedings of The 42nd Annual Conference of IEEE Industrial Electronics Society (IECON 2016), Fortezza da Basso, Firenze (Florence), Italy, (2016), pp. $4762-4766$.

[14] Y. Wiseman, "Compression Scheme for RFID Equipment", In Proceedings of IEEE International Conference on Electro Information Technology (EIT 2016), Grand Forks, North Dakota, USA, (2016), pp. 387-392.

[15] G. Fritz, V. Beroulle, M. D. Nguyen, O. Aktouf and I. Parissis, "Read-Error-Rate evaluation for RFID system on-line testing", In 2010 IEEE 16th International Mixed-Signals, Sensors and Systems Test Workshop (IMS3TW), (2010), pp. 1-6.

[16] C. Gomez, J. Oller and J. Paradells, "Overview and evaluation of bluetooth low energy: An emerging low-power wireless technology”, Sensors Journal, MDPI, vol. 12, no. 9, (2012), pp. 11734-11753.

[17] L. Zheng, X. He, B. Samali and L. T. Yang, "An Algorithm for Accuracy Enhancement of License Plate Recognition", Journal of Computer and System Sciences, Elsevier, vol. 79, no. 2, (2013), pp. 245-255.

[18] Y. Wiseman, "Take a Picture of Your Tire!", Proceedings of IEEE Conference on Vehicular Electronics and Safety, Qingdao, ShanDong, China, (2010), pp. 151-156.

[19] Y. Wiseman, "The Effectiveness of JPEG Images Produced by a Standard Digital Camera to Detect Damaged Tyres", World Review of Intermodal Transportation Research, vol. 4, no. 1, (2013), pp. 23-36.

[20] Y. Wiseman, "Camera That Takes Pictures of Aircraft and Ground Vehicle Tires Can Save Lives", Journal of Electronic Imaging, published by SPIE and the Society for Imaging Science and Technology, vol. 22, no. 4, (2013).

[21] M. S. McCorquodale, S. M. Pernia, J. D. O'Day, G. Carichner, E. Marsman, N. Nguyen, S. Kubba, S. Nguyen, J. Kuhn and R. B. Brown, "A 0.5 -to- $480 \mathrm{MHz}$ self-referenced CMOS clock generator with $90 \mathrm{ppm}$ total frequency error and spread-spectrum capability", In proceedings of IEEE International Solid-State Circuits Conference, (ISSCC 2008), Digest of Technical Papers, San Francisco, CA, USA, (2008), pp. 350-619.

[22] A. M. A. Rushdi and M. H. Amashah, "Using variable-entered Karnaugh maps to produce compact parametric general solutions of Boolean equations", International Journal of Computer Mathematics, vol. 88, no. 15, (2011), pp. 3136-3149.

[23] M. E. Holder, “A modified Karnaugh map technique”, IEEE Transactions on Education, vol. 48, no. 1, (2005), pp. 206-207.

[24] Y. T. Hwang, J. F. Lin and M. H. Sheu, "Low-power pulse-triggered flip-flop design with conditional pulse-enhancement scheme", IEEE transactions on very large scale integration (VLSI) systems, vol. 20, no. 2, (2012), pp. 361-366.

[25] X. Wang and W. H. Robinson, "A low-power double edge-triggered flip-flop with transmission gates and clock gating", In 53rd IEEE International Midwest Symposium on Circuits and Systems (MWSCAS), (2010), pp. 205-208.

[26] A. Chen and K. Subprasom, "Analysis of regulation and policy of private toll roads in a build-operatetransfer scheme under demand uncertainty", Transportation Research Part A: Policy and Practice, vol. 41, no. 6, (2007), pp. 537-558.

[27] H. Yang and Q. Meng, "Highway pricing and capacity choice in a road network under a build-operatetransfer scheme", Transportation Research Part A: Policy and Practice, vol. 34, no. 3, (2000), pp. 207222.

[28] H. Yang and Q. Meng, "A note on "highway pricing and capacity choice in a road network under a build-operate-transfer scheme"', Transportation Research Part A: Policy and Practice, vol. 36, no. 7, (2002), pp. 659-663.

[29] I. Grinberg and Y. Wiseman, "Scalable Parallel Simulator for Vehicular Collision Detection", International Journal of Vehicle Systems Modelling and Testing, Inderscience, vol. 8, no. 2, (2013), pp. 119-144.

[30] I. Grinberg and Y. Wiseman, "Scalable Parallel Collision Detection Simulation", In Proceedings of Signal and Image Processing, sponsored by the International Association of Science and Technology for Development (IASTED), Honolulu, Hawaii, (2007), pp. 380-385.

[31] Y. Wiseman and Y. Giat, "Multi-modal passenger security in Israel", Multimodal Security in Passenger and Freight Transportation: Frameworks and Policy Applications, Edward Elgar Publishing Limited, Chapter 16, (2016), pp. 246-260. 
[32] J. Odeck and S. Bråthen, "On public attitudes toward implementation of toll roads - the case of Oslo toll ring”, Transport Policy, vol. 4, no. 2, (1997), pp. 73-83.

[33] Y. Wiseman, “A Pipeline Chip for Quasi Arithmetic Coding”, IEICE Transactions on Fundamentals of Electronics, Communications and Computer Sciences, Tokyo, Japan, vol. E84-A, no. 4, (2001), pp. 1034-1041.

[34] Y. Wiseman, K. Schwan and P. Widener, "Efficient End to End Data Exchange Using Configurable Compression", In Proceedings of The 24th IEEE Conference on Distributed Computing Systems (ICDCS 2004), Tokyo, Japan, (2004), pp. 228-235.

[35] Y. Wiseman, "Computerized Traffic Congestion Detection System", International Journal of Transportation and Logistics Management, Global Vision Schoolpublication, vol. 1, no. 1, (2017), pp. 18.

[36] Y. Wiseman, "Tool for Online Observing of Traffic Congestions", International Journal of Control and Automation, vol. 10, no. 6, (2017), pp. 27-34

[37] R. C. Carlson, I. Papamichail, M. Papageorgiou and A. Messmer, "Optimal mainstream traffic flow control of large-scale motorway networks", Transportation Research Part C: Emerging Technologies, vol. 18, no. 2, (2010), pp. 193-212.

[38] D. A. Hensher, "Climate change, enhanced greenhouse gas emissions and passenger transport-What can we do to make a difference?", Transportation Research Part D: Transport and Environment, vol. 13, no. 2, (2008), pp. 95-111.

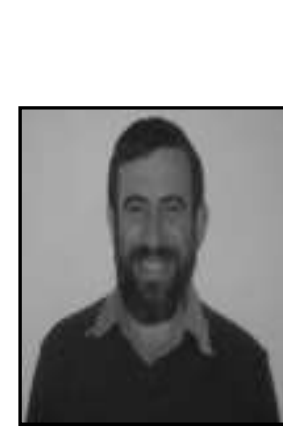

\section{Author}

Yair Wiseman, received the M.Sc. (summa cum laude) and Ph.D. degrees from Bar-Ilan University, the first Post-Doctoral degree with the Hebrew University of Jerusalem, and the second Post-Doctoral degree with the Georgia Institute of Technology.

His research interests include autonomous vehicles, intelligent transportation systems, embedded systems, real-time systems, computational transportation science, operating systems, and process scheduling.

Dr. Wiseman has authored two books. He is on the editorial board of several journals, a member of dozens of conference committees and a reviewer of many scholarly journals.

Dr. Wiseman has been teaching in many institutes including BarIlan University, The Hebrew University of Jerusalem and Israel Aircraft Industry and has been supervising many graduate students. An interesting point is that Albert Einstein is Dr. Wiseman's academic great-great-grandfather (i.e. the advisor of the advisor of the advisor of Dr. Wiseman's advisor).

Dr. Wiseman has collaborated with other partners and received research grants to run an active laboratory from inter alia Sun Microsystems, Intel, and Polak Foundation.

Dr. Wiseman is an international expert who has reviewed and evaluated several large projects of the European Union, Israel Science Foundation, Marie Skłodowska-Curie actions in Ireland, Ministry of Education and Science of Kazakhstan. His papers have been published in many venues around the world. 